\title{
Moral Competence in Nursing PRACTICE
}

\author{
Pantip Jormsri, Wipada Kunaviktikul, Shaké Ketefian and \\ Aranya Chaowalit
}

Key words: moral competence; nursing practice; nursing values; Thailand

This article presents the derivation of moral competence in nursing practice by identifying its attributes founded on Thai culture. In this process moral competence is formed and based on the Thai nursing value system, including personal, social and professional values. It is then defined and its three dimensions (moral perception, judgment and behavior) are also identified. Additionally, eight attributes as indicators of moral competence are identified and selected from three basic values. The eight attributes are loving kindness, compassion, sympathetic joy, equanimity, responsibility, discipline, honesty, and respect for human values, dignity and rights. All attributes are discussed by addressing the three moral dimensions in order to present how to deal with ethical issues in nursing practice. As a summary, a model of moral competence is presented to demonstrate moral competence in nursing practice in Thailand.

\section{Introduction}

The nursing profession requires increasing competence of its practitioners at all levels because competent nursing practice for the pursuit of health care is expected throughout society. Nursing practice-based competence must be emphasized and assessed among nurses. Zhang et al. defined competence as a person's capacity to meet a job's requirements by producing quality outcomes. ${ }^{1}$ According to Parsons, competence implies knowledge and the skills required in a profession, while also presuming the ability to apply that knowledge and those skills. Therefore, competence goes beyond simple knowing; it requires doing. ${ }^{2}$ Competence in actual practice is not the same as simply fulfilling educational requirements or maintaining a nursing license. ${ }^{3}$ Competence requires an up to date knowledge base and is manifested by demonstrating the ability to achieve desired outcomes through the performance of defined skills. ${ }^{4}$

Nursing competence is defined as the possession of basic nursing skills, including: (1) clinical competence - assessment and intervention skills, clinical judgment, and technical skills; (2) general competence - communication, critical thinking, and problem solving skills; and (3) moral competence - the individual's ability to live in

Address for correspondence: Pantip Jormsri, Faculty of Nursing, Chiang Mai University, Chiang Mai, 50200, Thailand. E-mail: pantip@chiangmai.ac.th 
a manner consistent with a personal moral code and role responsibilities. ${ }^{1,3,5}$ This is because nursing practice depends not only on technical knowledge and skills but also on values, beliefs and ethics, which play a significant role in shaping their decision making. Competence in ethics has also become part of the caring qualities of health care personnel, including nurses and physicians. ${ }^{6}$

\section{Moral competence in nursing practice}

Taylor stated that modern society requires moral competence of its nurses, charging them to be aware of moral competence in order to work through ethical issues encountered in their practice. ${ }^{5}$ Nurses who have skills in moral competence can be trusted to act in ways that advance the interest of patients. They can be accountable for the practice to themselves, the patients, the caregiving team and society. They also act as effective patient advocates and mediate ethical conflict among patients, significant others, health care team members, and other interested parties.

\section{Foundation of moral competence}

Moral competence in nursing practice requires familiarity with and commitment to nursing values. ${ }^{7}$ Wright ${ }^{8}$ mentioned that, in general, values influence ethical decision making in three ways: (1) values frame a problem and people view a problem on the basis of the values they bring to the situation; (2) values supply alternatives that humans consider as possible problem resolutions and are determined on the basis of the values they apply to their potential actions; and (3) values directing judgment or reasoning in resolving a problem are framed by what they wish to uphold or promote.

Since an understanding of ethical structure is a difficult and demanding task requiring intense self-awareness, a more specific framework is necessary to enable nurses to make numerous ethical decisions in their practice. ${ }^{9}$ A thoughtful reflection in terms of the contextual aspects of nursing in Thai culture is therefore necessary to develop the concept of moral competence along the lines of Thai nursing perspectives. Thai nursing values as constituting a value system need to be clarified first and then the concept of moral competence developed.

According to Fry, there are personal, cultural and professional values. ${ }^{10}$ In Veins's study, the source of nurse practitioners' values were shown to derive from familial and religious upbringing as well as from work experience in nursing. Nursing values influence nurses' views of goals, strategies and actions, and they can be considered as resources to guide nurses in engaging in ethically competent practice and in confronting contemporary ethical challenges. ${ }^{11}$ Naturally, nurses' life experiences, including the context of their life and practice, result in a way of knowing, and nursing knowledge is embedded in beliefs, values and traditions, religious and cultural observances, and other contextual layers of life. ${ }^{12}$

Based on all these concepts, the acquisition of nursing values is explored by means of personal, social and professional values. These three basic values are integrated into a Thai value system as a foundation for moral competence. Therefore, in developing the concept 'moral competence', meanings and attributes will be clarified for personal, professional and social values, as discussed below. 


\section{Personal values}

Personal values represent nurses' conceptions of what it means both to be and to act as a good nurse. From several surveys of nurses, ${ }^{13-15}$ it can be seen that they most often use personal values as resources in their ethical decision making. Blancett and Sullivan reported that families, religious figures and friends exerted the greatest influence on ethical decision making; these influences centered around personal values and beliefs. ${ }^{13}$ Determining, assessing and exploring personal values, and then identifying the impact of these values on nursing practice are essential for dealing with day to day ethical issues. ${ }^{16}$ This is because these values in the form of goals, attitudes, interests, feelings, convictions and beliefs embraced by nurses serve to guide their thinking, actions and interactions with patients. ${ }^{9,17}$

In order to determine the meaning of moral competence based on personal values, a sample of Thai nurses, including four nurse instructors and three nurse practitioners, were interviewed using semistructured questions, for example: What does moral competence mean in your opinion? What is moral competence in nursing practice? What are the indicators of moral competence? and How do nurses provide nursing practice based on moral competence? The results revealed their personal values acquired through their social and cultural interactions within nursing and wider society. These personal values are structurally essential components of the moral competence used as the major guideline in directing the nursing behaviors of the participants interviewed. Most of them viewed moral competence as an actual or potential ability residing in each person and serving as a resource on which nurses can draw for ethical working. This ability consists of thoughts, knowledge, skills, and so on, which capture the moral standpoint of each person. The nurses identified their values as indicators of moral competence, including loving kindness, compassion, sympathetic joy and equanimity, which tend to follow the Buddhist principle called the 'four sublime states of mind'. Additional indicators are responsibility, honesty and discipline.

\section{Social values}

Social values are multifaceted standards that guide conduct in a variety of ways, one of which is to lead an individual to take a particular position in a specific social situation. At the same time, social circumstances, including religion, culture, politics and economic considerations, impact on and form an individual's value system. Social values therefore temper human values. In Thai society, Buddhist social ethics takes root and has much significant meaning for contemporary life owing to the fact that a high proportion of Thai citizens are Buddhists. Over the centuries, Buddhist religious tradition has shaped human perception and values, and provided the moral norms pervading Thai culture. ${ }^{18}$ Thailand is a traditional society whose values have been articulated in terms of Buddhism. Buddhist teachings on personal conduct contain principles that could be reinterpreted and extended to form a social ethics theory. Buddhist social ethics would then become the guideline for action. ${ }^{19}$

In general, Buddhism provides guidelines for personal moral conduct such as compassion, generosity and mindfulness. ${ }^{19}$ Buddhist principles function for the Thai people as guidelines to distinguish between right and wrong, good and bad. Buddhists hold themselves responsible both for their own individual perfection and for the good of society, attaining both these goals via individual development and wellbeing as well as helpful social relationships. ${ }^{20}$ Occupying a central place in Buddhism, the four 
sublime states of mind - loving kindness, compassion, sympathetic joy and equanimity - can be viewed as values in nursing practice. The four bases of social harmony: giving, distribution and charity; kindly and beneficial words; rendering of services; and equality, impartiality and participation, are also necessary for maintaining good practice. ${ }^{20,21}$

Together with an analysis of Buddhist ethics literature related to nursing and health care, three health care service clients (one inpatient, one monk and one educator, as experts in Buddhism) were interviewed regarding moral competence in nursing practice by using semistructured questions, for example: How should moral competence be defined in nursing? What do you expect in nursing practice and is it related to moral competence? What can nurses perform to show moral competence in nursing practice? These results also supported adopting the four sublime states of mind as values constituting social values in nursing practice in Thai culture.

Not only Buddhist ethics, but also societal changes in health care delivery systems, such as advances in technology, scarcity or inappropriate allocation of resources, and increased complexity of client problems, affect nurses' perception, values, choices and actions in the pursuit of quality nursing care. ${ }^{22-25}$ Health care reform creates the need to restructure the system to incorporate values of equity, equality and respect for human dignity and patient rights. ${ }^{26}$ As a result, nurses must be aware of the revolutionary changes that shape the health care delivery system. For instance, the lack of just access and allocation of health care resources has caused nurses to advocate for change in order to protect individual patients from organizational exploitation and to influence the health and welfare of the wider community. ${ }^{27}$

In respect of both Buddhist ethics and societal issues related to the health care delivery system, there are certain values constituting social values such as the four sublime states of mind, the four bases of social harmony, the values of equity, justice and respect for human dignity, and patient rights. All social values are considered as a framework for identifying the indicators of moral competence because they influence day to day nursing practice, especially with regard to ethical decision making and behavior.

\section{Professional values}

Weis and Schank state that professional values are standards for action that provide a framework for evaluating nurse behavior. In practice, nurses make decisions that define and maintain a certain ethic. ${ }^{28}$ They contended that a professional code of ethics is a way of interpreting professional obligations: a statement of values and an assertion of goals. These professional values become the basis for standards of practice, producing a corresponding shift in professional identity and behavior. ${ }^{29}$

The Thai Nurses' Association Code of Ethics embodies a set of ideals, the interpretation and application of which are central to practice. The Code also provides guidelines for nursing practice throughout the country. It is considered a hallmark of professionalism; it states that a nurse has an obligation to individuals, to society and the country, to the profession, to nursing coworkers and workers in other fields, and to the self. ${ }^{30}$ Based on its significance, the Thai code of ethics was analyzed to identify professional nursing values. Professional values are therefore conceptualized as the fundamental values providing direction for nursing practice. These are addressed by Piyasirisilp: ${ }^{31}$ 
- Respect for the value of life: accepting that everyone has equal rights, and demonstrating no gender, age, religious or national discrimination.

- Faith in living: accepting that everyone has abilities and needs.

- Respect for human dignity: accepting that everyone has a right to privacy, honoring differences in needs, behaviors, goals, etc.

Self: responsibilities, respect, development, truth loving, etc.

Others: honesty, liberty, helpfulness, etc.

- Respect for aesthetics: accepting standards of goodness, beauty, art, happiness, quality, etc. (translated from the Thai by author PJ).

In addition, professional nursing values in Thailand derive from the Professional Nursing and Midwifery Act. $^{32}$ On analyzing the ethical aspects of the Act, Pornwattanakul identified eight attributes accepted as values that provide guidelines for nursing practice: faithfulness, politeness, kindness, respect for human dignity, discipline, unity, responsibility and devotion. ${ }^{33}$

Some of the professional values identified from the code of ethics and the Act ${ }^{31,33}$ can be identified as attributes of moral competence because these professional values are accepted as guidance for nursing practice in Thailand.

\section{Definition and dimensions of moral competence}

Before clarifying and defining its eight attributes in particular, moral competence needs to be defined, together with its appropriateness in nursing practice. Based on the outcome of interviews with the nurse educators, nurse practitioners and health care service clients, and the review of a considerable amount of literature, moral competence can here be defined as the ability or capacity of persons to recognize their feelings as they influence what is good or bad in particular situations, and then to reflect on these feelings, to make their decision, and to act in ways that bring about the highest level of benefit for patients. In consideration of its definition, moral competence is seen as a combination of three dimensions: (1) moral perception as an affective dimension requires the individual's awareness of values and the expression of those values in clearly communicated messages about the same; (2) moral judgment as a cognitive dimension entailing the individual's choice of one value over another based on logical reasoning and critical thinking; and (3) moral behavior as a behavioral dimension involving the individual's application of values to action by being willing to receive public affirmation for the choice, and consistent repetition of the same.

\section{Attributes of moral competence}

With respect to values in Thai nursing practice, eight attributes can be selected from the nursing values derived from personal, social and professional values. These are later considered as the attributes or indicators of moral competence in nursing practice. They are: loving kindness, compassion, sympathetic joy, equanimity, responsibility, discipline, honesty, and respect for human values, dignity and rights.

Values and beliefs are influenced by culture, religion and lived experience. The nursing values that are considered to be attributes of moral competence thus inevitably conform to Buddhist ethics in Thai society. With the effects of Thai culture and Buddhist ethics, four attributes of moral competence tend to follow the Buddhist 
principle called the 'four sublime states of mind', which are loving kindness, compassion, sympathetic joy and equanimity. This is because Buddhism influences the way of living for Thai people and Buddhist values are profoundly integrated into all aspects of Thai society. ${ }^{34}$

At present it is questionable whether a single moral framework is sufficient to account for the morality of all individuals in all cultures. Huebner and Garrod ${ }^{35}$ commented that western moral reasoning theories are inadequate to account for moral reasoning in Buddhist culture, especially when ethical decision making in Buddhist culture is necessarily based on an analysis of Buddhist philosophy. It is therefore appropriate to begin to identify the attributes as central elements of moral competence in Thai culture based on the Buddhist religion.

In addition, the attributes based on Thai culture have to be clarified and defined in all three dimensions of moral competence: the affective dimension as moral perception, the cognitive dimension as moral judgment, and the behavioral dimension as moral behavior. This is because all dimensions can present what and how nurses express moral competence in nursing practice. As a result, each attribute of moral competence herein represents appropriate Thai nursing values and three important dimensions of moral competence in nursing practice.

\section{Loving kindness}

Loving kindness is the human expression of a generous character. It means to exhibit an all-embracing kindness (affective dimension), the desire to make others happy as opposed to causing hatred or suffering (cognitive dimension), and to generate only friendliness among all living things (behavioral dimension). This value is important for nurses because it means their desire and intention to care for patients. Nurses also need to understand patients' circumstances. Because of their good intentions, they take care of patients with loving kindness. ${ }^{36}$

Loving kindness has usually been studied together with compassion. In Thailand both concepts have been studied in relation to nursing ethics because they are important virtues based on the Buddhist religion. They are presented as significant ethical characteristics that are evaluated in nurses and nursing students. ${ }^{33,37-39}$ In her study, An analysis of the ethics of care: a case study of the Thai nursing profession, Rawekchome ${ }^{37}$ interviewed a number of nurses and found that the principles of loving kindness and compassion were the most important concerns in their ethic of care. In Buddhist thought, loving kindness is the most significant value for nursing care, especially for a good relationship between nurses and patients. It is related to the wellbeing of patients and significant others. That is, when nurses exhibit loving kindness with good intention, patients will benefit most. ${ }^{21,40}$ Loving kindness must therefore be adopted as an attribute of moral competence in nursing practice.

\section{Compassion}

Compassion is well known as a nursing value. It means to have pity for the suffering of others (affective dimension), and the desire to free sufferers from their pain (cognitive dimension). It also avoids harmful actions and shows pity for the human plight (behavioral dimension). Loving kindness and compassion are always mutual in nursing care. ${ }^{37}$ Compassion occurs when nurses see patients' suffering or know for whom they need to care. Nurses then desire to free patients from their pain by 
avoiding harmful actions. It can therefore be concluded that, in daily nursing practice, nurses perceive patients' suffering, support their feelings, and then perform nursing roles in a spirit of loving kindness and compassion in order to free them from their suffering. ${ }^{21,36}$ As cited in Lundberg and Boonprasabhai, ${ }^{41}$ Thai senior women undergraduate nursing students mentioned compassion as one of the six categories that described their interpretations of good nursing care.

According to Dietze and Orb, ${ }^{42}$ nurse scholars identify compassion as one of the central characteristics that nurses are expected to possess. Compassion is often considered as an essential component of nursing care; it is not simply a natural response to suffering, but more of a moral choice. Similarly, Tuckett ${ }^{43}$ stated that compassion is a moral virtue that gives context and direction to nurses' decisions and actions and, as such, promotes excellence in nursing practice. Jull ${ }^{44}$ stated that compassion is not only a feeling but also a moral virtue that requires nurses to take action in the presence of suffering. Consequently, it is reasonable to include compassion as a source of the moral competence that nurses are expected to possess and exhibit in their practice.

\section{Sympathetic joy}

Sympathetic joy for human happiness is an important concern in nursing practice. It includes feeling appreciative pleasure (affective dimension) when seeing others happy and when seeing others do good actions or attain success and advancement. It also means responding gladly (cognitive dimension) by providing help and supporting others as they struggle to do good (behavioral dimension). Based on the four sublime states of mind, sympathetic joy is one of the important nursing practice values. This is because it maintains and encourages good relationships between nurses and others, and creates a happy and peaceful health care environment. With respect to sympathetic joy, nurses express pleasure when seeing patients happy, and when they succeed in obtaining medical care or regain their health. Nurses co-operate with nursing team members and other health care providers by providing sincere help and support, and they feel happy when seeing others carrying out good actions or attaining success and advancement.

Although it is generally known as a value in Thai Buddhist ethics and as a concern in nursing practice, sympathetic joy has not been studied in nursing ethics; it may be found in part in loving kindness and compassion. In the western nursing ethics literature the term 'sympathy' has been widely studied. Olsen ${ }^{45}$ stated that sympathy in nursing is often equated with empathy in that the two are strongly related to each other. They are also frequently used interchangeably, even though they are not exactly the same. Some theorists believe that sympathy has been devalued as sentimentality, while empathy has been promoted as a more professional approach. ${ }^{44}$ Sympathy is inclusive and often used interchangeably with compassion in nursing; ${ }^{42,44}$ however, sympathetic joy and compassion are distinguished from each other because their definitions show them to be distinct attributes of moral competence in nursing practice.

\section{Equanimity}

Equanimity means the acceptance that things are as they are (affective dimension). It is to understand (cognitive dimension) that everyone experiences good and evil in accordance with their actions without prejudice or preference, but in the light of Karma or the law of cause and effect. Equanimity builds up the habit of dealing with 
everything from the point of view of right or wrong by means of principles, reasons and equity (behavioral dimension). It ultimately leads to even mindedness based on insight into the nature of things. According to Tongprateep and Soowit, ${ }^{21}$ equanimity is considered as a very important virtue for nurses. In daily clinical practice they have to control their feelings and understand each patient's situation. They also have to accept their limitations in wishing to save patients' lives. If nurses have equanimity, they will not be sad when patients cannot get well or some of them die, because nurses understand patients' experience according to the cause of their condition. In nursing ethics, equanimity has been studied little, although it is a significant value for nurses, especially in Thai culture.

To have equanimity in their practice, nurses see things as they are with minds that are even, steady, firm and fair. They also need to understand that all beings experience good and evil in accordance with the universal law of cause and effect called Karma. ${ }^{36}$ Equanimity is therefore considered as a basis for showing loving kindness, compassion and sympathetic joy. Some Buddhist scholars have stated that it is very hard to practise equanimity. ${ }^{21}$

In their day to day practice, nurses always have opportunities to use the four sublime states of mind - loving kindness, compassion, sympathetic joy and equanimity - in caring for patients, especially when nursing is seen in the light of the law of cause and effect, which is associated with lives and society. Nursing helps and makes humans happy, and nurses provide care based on a holistic approach that considers physical, mental, social and spiritual aspects. ${ }^{46}$ The nursing profession in Thailand is thus inevitably related to Buddhist ethics; in other words, Thai nurses always adopt Buddhist principles as a framework or a guideline in their practice.

The remaining attributes - responsibility, discipline, honesty and respect for human values, dignity and rights - can be found in a number of nursing ethics studies carried out in Thailand. ${ }^{33,38,39,47-50}$ All these studies have evaluated the ethical characteristics of nurses, nurse instructors and nursing students. Ethical characteristics are often composed of honesty, loving kindness/compassion, discipline, responsibility, gentleness, respect for human beings, unanimity, devotion, sacrifice, and so on. These have thus been accepted as important characteristics that nurses have to show in their practice. These attributes have also been studied in western nursing ethics. The development of moral competence needs thoughtful reflection in terms of contextual aspects of nursing in Thai culture. It is derived from Thai nursing values, so the last four attributes are specifically clarified and defined below.

\section{Responsibility}

Responsibility is the nursing value that expresses the prompt and faithful discharge of duty (affective dimension). It means that minor mistakes are quickly recognized and corrected (cognitive dimension), that actions are taken in emergencies, and that others are called on for assistance when necessary (behavioral dimension).

In the nursing literature responsibility is used with a variety of meanings. One of the responsibilities of the nursing profession is to promote health, although health may be variously defined depending on the philosophical and theoretical perspectives guiding the practice. According to the Thai Nurses' Association code of ethics, ${ }^{30}$ nurses have to take responsibility for several groups such as clients, the nursing profession, society and themselves. Most studies in nursing ethics carried out 
in Thailand have addressed the responsibility of nurses as being based on this concept. In her study regarding the ethical image of the nursing profession, Saungam-Aium ${ }^{51}$ believed that this is defined with respect to nurses' responsibilities towards patients, colleagues and the profession.

\section{Discipline}

Discipline as a nursing value is the ability of nurses to be aware and to organize their life for personal growth (affective and cognitive dimensions). They can then control and conduct themselves properly in the social environment by performing helpful rather than exploitative acts (behavioral dimension).

Discipline is an important nursing value in Thailand because it is always found in studies of the ethical behaviors of Thai nurses and nursing students. Wangpetch ${ }^{50}$ studied the moral behavior of nurses related to the Professional Nursing and Midwifery Act. ${ }^{32}$ The results showed that $95.1 \%$ of nurses exhibited a high level of moral behavior with respect to discipline. Likewise, Pornwattanakul's study ${ }^{33}$ revealed that discipline was an ethical behavior that nurses accepted to a high degree.

\section{Honesty}

Honesty focuses on human respect that upholds the truth, avoids deceit and strives to be sincere with others (affective and cognitive dimensions) by considering how this may be achieved and that being honest leads to right or good action. Honesty also includes both clearing up any misunderstandings when they occur, and avoiding gossip, back-biting, harsh words and idle speech (behavioral dimension).

It is generally known that honesty is crucial to personal integrity, not only in Buddhist thought but also according to universal beliefs. Thus honesty in nursing actions is an ethical behavior that nurses must perform, especially when they face choices of actions that can be either honest or dishonest. According to Suwanawong's pediatric study, ${ }^{52}$ it was accepted by both doctors and nurses that they had a duty to give truthful information to the parents of their patients.

\section{Respect for human values, dignity and rights}

Respect for human values, dignity and rights is important for nursing values. It can be defined as seeing human beings as neighbors and co-inhabitants of the world (affective dimension), and as thinking about them as both equal and unique (cognitive dimension). Moreover, respect for persons is implemented in two ways (behavioral dimension): at the level of individual autonomy and self-determination, and at the level of respect for individuals as members of the human community in which everyone is interdependent and interconnected.

The concept of respect permeates nursing practice at the macro level of planning and decision making and at the level of the day to day details of nursing engagement with patients. Nursing care without respect tends to dehumanize patients. ${ }^{53}$ Chaleawsak ${ }^{54}$ stated that advocacy/protecting patients' rights was one method that nurses used to resolve or cope with ethical dilemmas. In respect of this method of protecting patients' rights, the nurses studied dealt with ethical dilemmas based on patientcentered management. Human dignity is a concept used frequently in nursing and is deemed important in maintaining or promoting patients' health. ${ }^{55}$ Tiwattananon ${ }^{56}$ stated that there must be respect for people's dignity and humanity because everyone has their own values. 


\section{Model of moral competence}

Moral competence consists of attributes derived from personal, social and professional values. The information obtained from the interviews of nurse educators and nurse practitioners can be accepted as personal values. For social values, although the Buddhist religion is the main source, the interviews with health care service clients as Buddhists are also important. In addition, the Thai Nurses' Association code of ethics ${ }^{30}$ and the Thai Professional Nursing and Midwifery Act $^{32}$ are also referred to as a basis for identifying professional values. The comprehensive review of related literature was very helpful for clarifying and defining all the attributes in accordance with nursing practice in Thailand.

Based on its foundation, the concept of moral competence is summarized as a model in Figure 1. This model demonstrates all sources of developing moral competence as nursing values or a value system in the nursing profession. It presents the three dimensions and eight attributes of moral competence. This model therefore represents a conceptualization of moral competence in nursing practice.

\section{Summary}

In organizing the Thai nursing value system as a foundation for the development of moral competence in nursing, moral competence is derived from three basic values: personal, social and professional. According to its definition, moral competence can be analyzed and specified as having three dimensions: moral perception, moral judgment and moral behavior. Moral competence is, additionally, comprised of eight attributes derived from all three aspects of the Thai nursing value system. The dimensions and

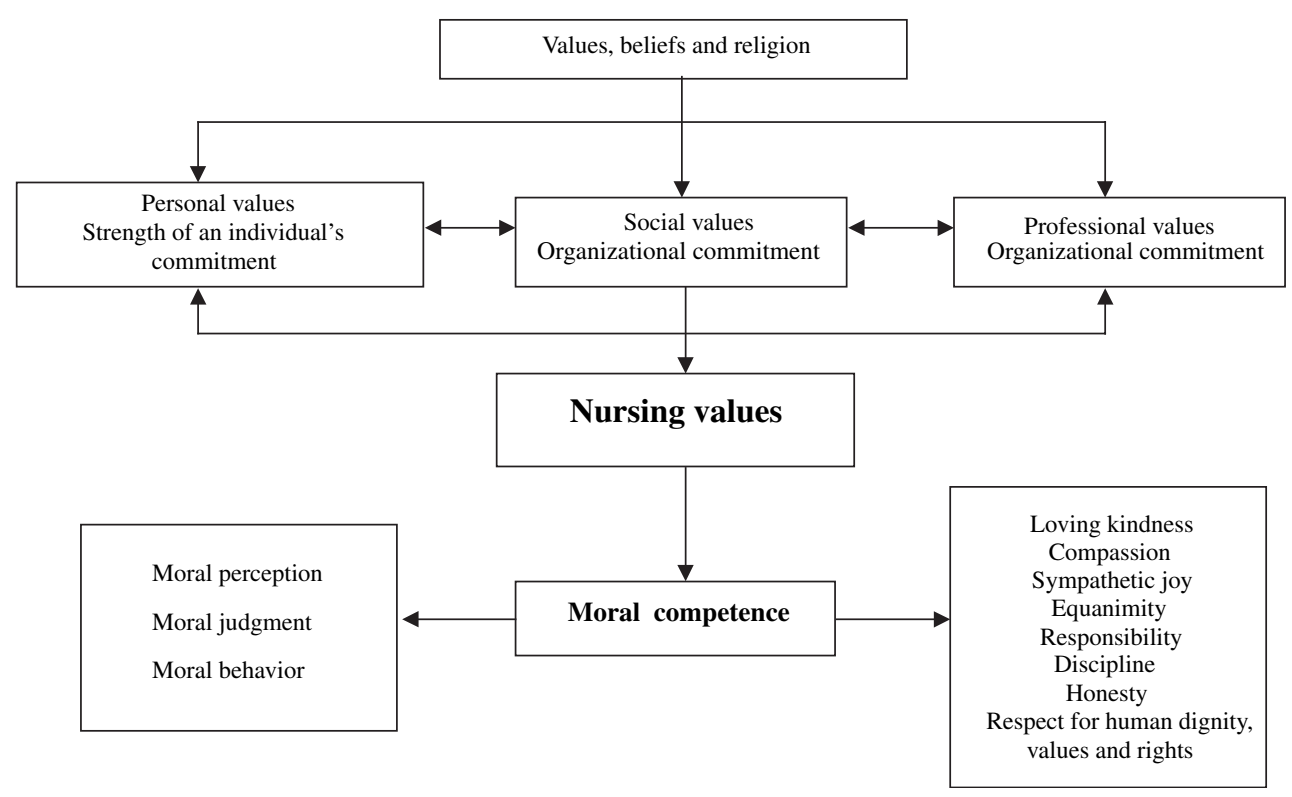

Figure 1 Moral competence in nursing practice in Thailand 
attributes of moral competence are clarified in this article in order to point out how nurses can deal with ethical issues in their practice in a culturally sensitive way. The moral competence model (Figure 1) is helpful for summarizing and demonstrating the development of the concept of 'moral competence'. In this article moral competence is conceptualized as the ability of nurses to deal with ethical issues in their practice. It thus needs to be considered for the resolution of ethical problems and the moral choices justifying their rationales. The model can be considered as the theoretical rationale and it was later used as a framework for developing a scale for measuring moral competence in nursing practice in Thailand.

Pantip Jormsri and Wipada Kunaviktikul, Chiang Mai University, Chiag Mai, Thailand. Shaké Ketefian, University of Michigan, Ann Arbor, MI, USA. Aranya Chaowalit, Prince of Songkla University, Songkla, Thailand.

\section{References}

${ }^{1}$ Zhang Z, Luk W, Arther D, Wong T. Nursing competencies: personal characteristics contributing to effective nursing performance. J Adv Nurs 2001; 33: 467-74.

2 Parsons RD. The ethics of professional practice. Boston, MA: Allyn and Bacon, 2000.

${ }^{3}$ Lenburg CB. Promoting competence through critical self-reflection - a portfolio development: the inside evaluator and the outside context. Tennessee Nurse 2000; 63(3): 11,14-15.

${ }^{4}$ LaDuke S. Ethical issues in pain management. Crit Care Nurs Clin North Am 2002; 14: 165-70.

5 Taylor C. Rethinking nursing's basic competencies. J Nurs Care Qual 1995; 9(4): 1-13.

${ }^{6}$ Savulescu J, Crisp R, Fulford KW, Hope T. Evaluating ethics competence in medical education. J Med Ethics 1999; 25: 367-74.

7 Scanlon C, Glover J. A professional code of ethics: providing a moral compass for turbulent times. Oncol Nurs Forum 1995; 22: 1515-21.

8 Wright RA. Human values in health care: the practice of ethics. New York: McGraw-Hill, 1987.

9 Moss MT. Principles, values, and ethics set the stage of managed care nursing. Nurs Econ 1995; 13: 276-84.

${ }^{10}$ Fry ST. Ethics in nursing practice: a guide to ethical decision making. Geneva: International Council of Nurses, 1994.

11 Veins DC. The moral reasoning of nurse practitioners [Dissertation]. San Diego, CA: University of San Diego, 1991.

12 Will CI. Knowledge in nursing: contemplating life experience. Can J Nurs Res 2001; 3(3): 107-16.

13 Blancett S, Sullivan P. Ethics survey results. J Nurs Adm 1993; 23(3): 9-13.

14 Borawski DB. Resources used by nurse administrators in ethical decision-making. J Nurs Adm 1994; 24(3): 17-22.

15 Sietsema M, Spradley B. Ethics and administrative decision making. J Nurs Adm 1987; 17(4): $28-32$.

16 Scanlon C, Fleming C. Ethical issues in caring for the patient with advanced cancer. Nurs Clin North Am 1989; 24: 977-86.

17 Fagermoen MS. Professional identity: values embedded in meaningful nursing practice. J Adv Nurs 1997; 25: 434-41.

18 Ratanakul P. Bioethics in Thailand: the struggle for Buddhist solutions. J Med Philos 1988; 13: 301-12.

19 Puntarigvivat T. Toward a Buddhist social ethics: the case of Thailand. Cross Currents 1998; 48(3). 15 December, 2002. Retrieved on 31 July, 2005, from: http://www.crosscurrents.org/ buddhistethics.htm 
${ }^{20}$ Rajavaramuni (Thepwethi) Buddha. Foundations of Buddhist social ethics. In: Sizernore RF, Swearer DK eds. Ethics, wealth, and salvation: a study in Buddhist social ethics. Columbia, SC: University of South Carolina Press, 1990: 29-53.

21 Tongprateep T, Soowit B. Brahma vihara: the wisdom of nursing. In: Tassanee T, Noot T, Nonglak N et al. eds. Buddhism for nursing. Bangkok: Eak Pim Thai Press, 2002: 55-68 (in Thai).

22 Ericksen J. Step to ethical reasoning. Can Nurse 1989; 85(7): 23-24.

23 Rodney P, Starzomski R. Constraints on the moral agency of nurses. Can Nurse 1993; 89(9): 23-26.

24 Dierckx de Casterlé BD, Janssen PJ. The relationship between education and ethical behavior of nursing students. West J Nurs Res 1996; 8: 330-50.

25 Hamric AB. Moral distress in everyday ethics. Nurs Outlook 2000; 4: 199-201.

${ }^{26}$ Ketefian S, Phancharoenworakul K, Yunibhand J. Research priorities in nursing ethics for Thailand. Thai J Nurs Res 2001; 5: 111-17 (in Thai).

27 Badzek LA, Mitchell K, Marra SE, Bower MM. Administrative ethics and confidentiality privacy issues. Online Journal of Issues in Nursing 31 Dec, 1998. Retrieved on 31 July, 2005, from: http://www.nursingworld.org/ojin/topic8/topic8_2.htm

28 Weis D, Schank MJ. An instrument to measure professional nursing values. Image J Nurs Sch 2000; 32: 201-204.

29 Weis D, Schank MJ. Professional values and empowerment: a role for continuing education. J Contin Educ Nurs 1991; 22(2): 50-53.

30 Thai Nurses' Association code of ethics. Bangkok: Thai Nurses' Asssociation, 1985 (in Thai).

31 Piyasirisilp S. Relationships between professional nursing values, academic achievement and the ability to make decisions in nursing ethical dilemmas of nursing students [Thesis]. Bangkok: Chulalongkorn University, 1996 (in Thai).

32 Nursing Council of Thailand. The Professional Nursing and Midwifery Act BE 2528 (revision 2540). Bangkok: Nursing Council of Thailand, 1997 (in Thai).

33 Pornwattanakul S. Professional nurse ethical behaviors in hospitals in Nonthaburi Province indicated in the Professional Nursing and Midwifery Act BE 2528 [Thesis]. Bangkok: Mahidol University, 1999 (in Thai).

34 Tongprateep T, Tipseankhum N. Dhama for nursing. In: Tassanee T, Noot T, Nonglak N et al. eds. Buddhism for nursing. Bangkok, Thailand: Eak Pim Thai Press, 2002: 1-15 (in Thai).

35 Huebner A, Garrod A. Moral reasoning in a Karmic world. Human Development 1991; 34: 341-52.

36 Thepwethi Buddha. Buddhist dictionary, seventh edition. Bangkok: Chulalongkorn University Press, 1992 (in Thai).

37 Rawekchome P. An analysis of the ethics of care: a case study of the Thai nursing profession [Thesis]. Bangkok: Mahidol University, 1998 (in Thai).

38 Sattayatham C, Vaivong N. Scholastic achievement and ethics of student nurses in Boromarajunani College of Nursing, Srithunya. Bangkok: Srithunya Nursing College, 1996 (in Thai).

39 Suwanpatikorn K. A comparison of moral values of nursing students being taught by the value clarification method and the traditional teaching method [Thesis]. Bangkok: Mahidol University, 1991 (in Thai).

40 Tongprateep T. Loving-kindness and compassion: moral principles for nurses. In: Tassanee T, Noot T, Nonglak N et al. eds. Buddhism for nursing. Bangkok, Thailand: Eak Pim Thai Press, 2002: 69-83 (in Thai).

41 Lundberg PC, Boonprasabhai K. Meanings of good nursing care among Thai female last-year undergraduate nursing students. J Adv Nurs 2001; 34: 35-42.

42 Dietze E, Orb A. Compassionate care: a moral dimension of nursing. Nurs Inquiry 2000; 7: 166-74.

43 Tuckett AG. An ethic of the fitting: a conceptual framework for nursing practice. Nurs Inquiry 1998; 5: 220-27.

44 Jull A. Compassion: a concept exploration. Nursing Praxis in New Zealand 2001; 17(1): 16-23. 
45 Olsen DP. Development of an instrument to measure the cognitive structure used to understand personhood in patients. Nurs Res 1997; 46(2): 78-84.

46 Tongprateep T, Nareuwat N. Religion: a basis for nursing practice. In: Tassanee T, Noot T, Nonglak N et al. eds. Buddhism for nursing. Bangkok: Eak Pim Thai Press, 2002: 17-31 (in Thai).

47 Chamnanpood C. Moral behaviors of nursing students according to professional codes of ethics: in the nursing colleges under the Nursing College Division Office of the Permanent Secretary, Ministry of Public Health. Journal of Rachaburi Nursing College 1994; 6(1): 1-7 (in Thai).

48 Nikomrat R, Yomsriken S, Tungpradith S, Pukkesorn U. A study of moral characteristics of nursing students, Sawanpracharak Nursing College. Thai Nurs J 1989; 38(1): 19-26 (in Thai).

49 Ratanapanya D, Charonyuth C, Rungreungkul N, Chaowalitnithikul U. Ethical characteristics of nurse instructors at Mahidol University. Bangkok: Mahidol University, 1997 (in Thai).

50 Wangpetch A. Moral behavior of nurses under the act on the nursing and midwifery profession BE 2528: an empirical study for Siriraj Hospital [Thesis]. Bangkok, Thailand: Mahidol University, 2002 (in Thai).

51 Saungam-Aium N. A study of the ethical image of the nursing profession according to the views of nurses and clients in hospitals under the Department of Medical Services, Ministry of Public Health, in the metropolitan area [Thesis]. Bangkok: Mahidol University, 2003 (in Thai).

52 Suwanawong P. Ethical issues in pediatrics: ethics, Buddhist ethics and medical practicability in Thai society [Thesis]. Bangkok: Mahidol University, 1998 (in Thai).

53 McGree P. The concept of respect in nursing. Br J Nurs 1994; 3: 681-84.

54 Chaleawsak K. Ethical dilemmas experienced by nurses in providing care for terminally ill patients [Thesis]. Songkla: Prince of Songkla University, 2001 (in Thai).

55 Haddock J. Towards further clarification of the concept 'dignity'. J Adv Nurs 1996; 24: 924-31.

56 Tiwattananon S. The right of the elderly to die: ethical problems [Thesis]. Bangkok: Mahidol University, 1997 (in Thai). 
Copyright of Nursing Ethics is the property of Arnold Publishers. The copyright in an individual article may be maintained by the author in certain cases. Content may not be copied or emailed to multiple sites or posted to a listserv without the copyright holder's express written permission. However, users may print, download, or email articles for individual use. 